\title{
Log-supermodularity of weight functions and the loading monotonicity of weighted insurance premiums
}

\author{
Hristo S. Sendov ${ }^{a}$, Ying $\mathrm{Wang}^{b}$, and Ričardas Zitikis ${ }^{a, *}$ \\ ${ }^{a}$ Department of Statistical and Actuarial Sciences, University of Western Ontario, London, \\ Ontario N6A 5B\%, Canada \\ ${ }^{b}$ Department of Applied Mathematics, University of Western Ontario, London, \\ Ontario N6A 5B\%, Canada
}

(E-mails: hssendov@stats.uwo.ca, ywang767@uwo.ca, zitikis@stats.uwo.ca)

\begin{abstract}
The paper is motivated by a problem concerning the monotonicity of insurance premiums with respect to their loading parameter: the larger the parameter, the larger the insurance premium is expected to be. This property, usually called loading monotonicity, is satisfied by premiums that appear in the literature. The increased interest in constructing new insurance premiums has raised a question as to what weight functions would produce loadingmonotonic premiums. In this paper we demonstrate a decisive role of log-supermodularity in answering this question. As a consequence, we establish - at a stroke - the loading monotonicity of a number of well-known insurance premiums and offer a host of further weight functions, and consequently of premiums, thus illustrating the power of the herein suggested methodology for constructing loading-monotonic insurance premiums.
\end{abstract}

\section{JEL Classification:}

C02 - Mathematical Methods

C44 - Statistical Decision Theory; Operations Research

C51 - Model Construction and Estimation

D81 - Criteria for Decision-Making under Risk and Uncertainty

Keywords and phrases: Insurance premium; Weighted premium; Weighted distribution; Logsupermodularity; Supermodularity; Submodularity; Monotonicity, Esscher premium; Conditional tail expectation; Kamps premium; Wang premium; Distortion premium; Chebyshev's inequality; Decision under uncertainty.

${ }^{*}$ Corresponding author: tel.: +1 519432 7370; fax.: +1 519661 3813; e-mail: zitikis@stats.uwo.ca 


\section{INTRODUCTION}

Let $(\Omega, \mathcal{A}, \mathbf{P})$ be a probability triplet. The integral $\mathbf{E}[X]=\int_{\Omega} X(\omega) \mathbf{P}(d \omega)$ is known in the statistical literature as the expectation (or mean) of the 'random variable' $X: \Omega \rightarrow \mathbf{R}$, and also known in the actuarial literature as the net premium of the 'loss variable' $X$. In the latter case, $X$ usually takes on non-negative values. In order to avoid mathematical trivialities of constant worry, throughout this paper except when explicitly noted otherwise, we work with positive random variables (i.e., $X>0$ almost surely) and denote their class by $\mathcal{X}_{+}$.

The net premium $\mathbf{E}[X]$ is the very minimum that the insurer needs to charge customers for agreeing to accept their risks and have a chance to remain solvent. In fact, in order to meet various financial obligations, the insurer charges a larger amount; denote it by $H[X]$. This defines a functional $H: \mathcal{X}_{+} \rightarrow[0, \infty]$, called premium calculation principle or, simply, premium. Any premium $H$ such that $H[X] \geq \mathbf{E}[X]$ for all $X \in \mathcal{X}_{+}$is called non-negatively loaded or, simply, loaded. Loaded premiums are constructed using various actuarial considerations and mathematical techniques, and we shall next briefly discuss two of them.

First and arguably one of the oldest general techniques for constructing loaded premiums is based on the fact that the net premium $\mathbf{E}[X]$ can be written - using the Fubini theorem or the integration by parts formula - as the integral $\int_{0}^{\infty} \mathbf{P}[X>x] d x$. (Recall that $X \in \mathcal{X}_{+}$; for real-valued $X$, we would have a Choquet integral; see Denneberg (1994).) Choose any function $g:[0,1] \rightarrow[0, \infty)$ such that $g(s) \geq s$ for all $0 \leq s \leq 1$ and modify the above integral into $\int_{0}^{\infty} g(\mathbf{P}[X>x]) d x$, which is a loaded premium, known in the actuarial literature as the distortion, or Wang's, premium (e.g., Denuit et al., 2005). The function $g:[0,1] \rightarrow[0, \infty)$ is called the distortion function and usually depends on a parameter, called the distortion parameter, which governs the amount of loading contained in the distortion premium.

The second and fairly recent avenue for constructing loaded premiums has been suggested by Furman and Zitikis (2008). Just like in the case of the distortion premium, the construction starts with the net premium $\mathbf{E}[X]$ but this time transforming the integrator $\mathbf{P}(d \omega)$ with a 'weight' function $w:[0, \infty) \rightarrow[0, \infty)$. This approach gives a new probability measure $\mathbf{P}_{w}(d \omega)=w(X(\omega)) \mathbf{P}(d \omega) / \mathbf{E}[w(X)]$, assuming of course that the 'normalizing' expectation $\mathbf{E}[w(X)]$ is non-zero, that is, positive. The weighted premium is (Furman and Zitikis, 2008)

$$
H_{w}[X]=\int_{\Omega} X(\omega) \mathbf{P}_{w}(d \omega)
$$

In many special cases of $H_{w}[X]$ that we find in the literature (see, e.g., Furman and Zitikis, 2008, 2009), the weight function $w$ is indexed with a parameter, which we denote by $\lambda$. The parameter controls the amount of loading and is therefore called the loading parameter. 
After a re-parametrization if necessary, the loading parameter $\lambda$ can be assumed to be in $(0, \infty)$, with the limiting value of $H_{w}[X]$ when $\lambda \downarrow 0$ associated with the net premium $\mathbf{E}[X]$.

From now on, therefore, instead of $w(x)$ we work with weight functions $x \mapsto w(\lambda, x)$ indexed by $\lambda \in(0, \infty)$. It therefore becomes natural to start using the notation $H[\lambda, X]$ instead of the more cumbersome $H_{w(\lambda, \cdot)}[X]$. That is, let

$$
H[\lambda, X]=\frac{\mathbf{E}[X w(\lambda, X)]}{\mathbf{E}[w(\lambda, X)]}
$$

and denote $\Lambda[w, X]=\{\lambda \in(0, \infty): \mathbf{E}[X w(\lambda, X)]<\infty$ and $\mathbf{E}[w(\lambda, X)]>0\}$, which is usually an interval, finite or infinite, depending on the cumulative distribution function (cdf) $F$ of the loss variable $X$. From the intuitive point of view, we expect that the larger the parameter $\lambda$ is, the larger the value of $H[\lambda, X]$ is. This monotonicity property may not always hold as it depends on the weight function $x \mapsto w(\lambda, x)$. Later in this paper we shall specify conditions under which the loading monotonicity of $H[\lambda, X]$ holds.

A special case of the loading monotonicity property, and one of the basic requirements for $H[\lambda, X]$ to satisfy, is the aforementioned non-negative loading property:

$$
H[\lambda, X] \geq \mathbf{E}[X] \quad \text { for all }(\lambda, X) \in(0, \infty) \times \mathcal{X}_{+} .
$$

The property is satisfied whenever the weight function $x \mapsto w(\lambda, x)$ is non-decreasing, which can be infered from a classical result of Lehmann (1966) stating that the bound $\mathbf{E}[u(X) v(X)] \geq \mathbf{E}[u(X)] \mathbf{E}[v(X)]$ holds for all non-decreasing (Borel) functions $u$ and $v$ for which the expectations are well-defined and finite. In fact, this property is well-known in Mathematical Analysis under the name of Chebyshev's integral inequality (see, e.g., Pečarić et al., 1992; Mitrinović et al., 1993; and references therein) and has been widely utilized when solving a number of problems in Economics and Finance (see, e.g., Broll et al., 2010; Egozcue et al., 2010; and references therein) where non-negativity of the covariance $\operatorname{Cov}[u(X), v(X)]$ plays a pivotal role.

The following assumption now becomes natural, and we let it hold throughout the paper without explicitly mentioning it.

Assumption 1.1. For every (loading) parameter $\lambda>0$, the weight function $x \mapsto w(\lambda, x)$ is non-decreasing and non-negative, and we also assume that the function is Borel-measurable so that expectations are calculable.

The rest of the paper is organized as follows. In Section 2 we establish a fundamental for this paper result stating that log-supermodularity of the function $(\lambda, x) \mapsto w(\lambda, x)$ implies loading monotonicity of the premium $H[\lambda, X]$. In Section 3 we provide a set of parametric weight functions that either lead to known insurance premiums or to new ones, and we also verify log-supermodularity of the functions thus establishing loading monotonicity of the 
corresponding weighted premiums. In Section 4 we work out conditions under which loading monotonicity turns into loading strict-monotonicity. In Section 5 we specify conditions under which the function $\lambda \mapsto H[\lambda, X]$ is right-continuous, and even continuous. Some results of technical nature are relegated to Section 6 .

Given the above outline, one may wonder if our research of monotonicity and continuity of the function $\lambda \mapsto H[\lambda, X]$ has been driven by mathematical curiosity or actuarial considerations. The answer is 'both'. Originally, our interest was inspired by an insurance-related problem, which subsequently brought in a number of interesting mathematical issues.

To explain the original problem, assume that an insurer favors using $H[\lambda, X]$ in all inhouse premium calculations and thus wishes to convert into it all the other premiums $\pi[X]$ in use. Hence, for each such $\pi[X]$, the insurer wishes to know $\lambda$ such that $H[\lambda, X]=\pi[X]$. Depending on the form of $H[\lambda, X]$, there might be several values of $\lambda$ that give the equality $H[\lambda, X]=\pi[X]$, or there might be none such $\lambda$. If at least one $\lambda$ exists, then one may still wish to know whether this $\lambda$ is the only one or not. Answering such questions, naturally, relies on monotonicity and continuity properties of the function $\lambda \mapsto H[\lambda, X]$, and they in turn depend on those of the function $(\lambda, x) \mapsto w(\lambda, x)$ coupled with properties of the loss variable $X$. Sorting out these issues in detail is our goal in the present paper. In the context of the aforementioned distortion premium, the problem has been posed and justified from the actuarial point of view by Jones and Zitikis (2007).

\section{LOG-SUPERMODULARITY AND LOADING MONOTONICITY}

To formulate the main result of this section, which is Theorem 2.1 below, we need to recall some definitions. Function $(\lambda, x) \mapsto w(\lambda, x)$ is called log-supermodular if $(\lambda, x) \mapsto$ $\log (w(\lambda, x))$ is supermodular, which is equivalent to saying that $L(\lambda, x)=-\log (w(\lambda, x))$ is submodular. The function $(\lambda, x) \mapsto L(\lambda, x)$ is submodular if

$$
L\left(\theta, x_{1}\right)+L\left(\lambda, x_{2}\right) \leq L\left(\theta, x_{2}\right)+L\left(\lambda, x_{1}\right)
$$

whenever $\theta \leq \lambda$ and $x_{1} \leq x_{2}$.

The way we have here presented the definition of submodularity is to facilitate computations. The standard way is in the form of the bound $L(\boldsymbol{y} \wedge \boldsymbol{z})+L(\boldsymbol{y} \vee \boldsymbol{z}) \leq L(\boldsymbol{y})+L(\boldsymbol{z})$, where the minimum $\boldsymbol{y} \wedge \boldsymbol{z}$ and the maximum $\boldsymbol{y} \vee \boldsymbol{z}$ between the vectors $\boldsymbol{y}=\left(y_{1}, y_{2}\right)$ and $\boldsymbol{z}=\left(z_{1}, z_{2}\right)$ are taken coordinatewise. For details on submodular, supermodular, and other related functions, we refer to, for example, Fujishige (1991), Narayanan (1997), and Topkis (2001). A number of elementary ways for constructing submodular functions are listed in Table I on p. 312 of Topkis (1978). Note also that if the function $L$ is sufficiently smooth, then its submodularity is equivalent to the bound $\left(\partial^{2} / \partial \lambda \partial x\right) L(\lambda, x) \leq 0$ for all $(\lambda, x)$. We shall utilize this criterion frequently in this paper. 
The following theorem is a fundamental result in the context of the present paper.

Theorem 2.1. If $(\lambda, x) \mapsto w(\lambda, x)$ is log-supermodular, then $\lambda \mapsto H[\lambda, X]$ is non-decreasing.

To prepare for the proof of Theorem 2.1, we need to recall weighted distributions and some of their properties (see, e.g., Furman and Zitikis, 2008, and references therein). Thus, assume that we are dealing with a non-decreasing function $w$, indexed or not. Denote the cdf of $X \in \mathcal{X}_{+}$by $F$. The weighted cdf $F_{w}$ is defined by

$$
F_{w}(x)=\frac{\mathbf{E}[\mathbf{1}\{X \leq x\} w(X)]}{\mathbf{E}[w(X)]},
$$

where $1\{S\}$ is equal to 1 if statement $S$ is true and 0 otherwise. Note the equation $F_{w}(x)=$ $\int_{\Omega} \mathbf{1}\{X(\omega) \leq x\} \mathbf{P}_{w}(d \omega)$ connects $F_{w}$ and $\mathbf{P}_{w}$ in the same way as the original $F$ and $\mathbf{P}$ are connected by the usual definition of the cdf $F(x)=\mathbf{P}[X \leq x]$. The support of $F_{w}$ is $[0, \infty)$.

The importance of the weighted cdf in the current context is reflected by the fact that if $X_{w}$ is a random variable with the cdf $F_{w}$, then the weighted premium $H_{w}[X]$ is the mean $\mathbf{E}\left[X_{w}\right]$. Indeed, using the Fubini theorem, we have that

$$
H_{w}[X]=\int_{[0, \infty)} \frac{\mathbf{E}[\mathbf{1}\{X>x\} w(X)]}{\mathbf{E}[w(X)]} d x=\int_{[0, \infty)}\left(1-F_{w}(x)\right) d x=\mathbf{E}\left[X_{w}\right] .
$$

Equation (2.2) plays a major role in establishing Theorem 2.1 as well as some other results in later sections. The following properties are known (see, e.g., Furman and Zitikis, 2008, and references therein) and will be used a number of times in this paper:

- For any two non-decreasing and non-negative functions $u$ and $w$, we have

$$
F_{u w}=\left(F_{u}\right)_{w}=\left(F_{w}\right)_{u},
$$

where $F_{u w}$ is the weighted cdf corresponding to the product $u(x) w(x)$ of the two functions $u(x)$ and $w(x)$.

- For any non-decreasing and non-negative function $w$, we have $F_{w} \leq F$ and, consequently, for any non-decreasing and non-negative functions $w$ and $h$, we have

$$
F_{h w} \leq F_{w} \leq F
$$

Proof of Theorem 2.1. We start by showing that $L(\lambda, x)=-\log (w(\lambda, x))$ is submodular if and only if for every pair $\theta \leq \lambda$ there is a non-decreasing function $h=h_{\theta, \lambda}$ such that

$$
w(\lambda, x)=h(x) w(\theta, x) .
$$

(We do not know if this reformulation of log-supermodularity has been noted in the literature, but we find it invaluable in the context of the present paper.) Assuming for the time being that decomposition (2.5) holds, we next show how to utilize it for proving Theorem 2.1.

Fix any pair $\theta \leq \lambda$ and let $h$ be a non-decreasing function whose existence is postulated by decomposition (2.5). In view of bounds (2.4), we have $F_{w(\lambda, \cdot)} \leq F_{w(\theta, \cdot)}$. Using equations 
(2.2), we therefore have $\mathbf{E}\left[X_{w(\lambda, \cdot)}\right] \geq \mathbf{E}\left[X_{w(\theta, \cdot)}\right]$ and thus $H[\lambda, X] \geq H[\theta, X]$, which is the claim of Theorem 2.1. We are left to demonstrate the equivalence of submodularity of $L(\lambda, x)$ and the existence of a non-decreasing function $h$ such that decomposition (2.5) holds.

Fix any pair $\theta \leq \lambda$. The submodularity of $L(\lambda, x)$ implies that, for $x_{1} \leq x_{2}$,

$$
w\left(\theta, x_{1}\right) w\left(\lambda, x_{2}\right) \geq w\left(\theta, x_{2}\right) w\left(\lambda, x_{1}\right)
$$

Define $y_{\Delta}=\sup \{x \in[0, \infty): w(\Delta, x)=0\}$ for $\Delta \in\{\theta, \lambda\}$. If $y_{\theta}=-\infty$, that is, if $w(\theta, x)>0$ for all $x$, then the existence of the aforementioned function $h(x)$ is trivial. Otherwise, for every $x_{1}$ with $w\left(\theta, x_{1}\right)=0$, we choose $x_{2}>y_{\theta}$, and then bound (2.6) implies $w\left(\lambda, x_{1}\right)=0$ thus showing that $y_{\theta} \leq y_{\lambda}$. We can now define the sought after function $h$ as follows:

$$
h(x)= \begin{cases}0 & \text { when } x>0 \text { is such that } w(\theta, x)=0, \\ w(\lambda, x) / w(\theta, x) & \text { otherwise. }\end{cases}
$$

Note that $h$ is non-decreasing, as is implied by bound (2.6).

Conversely, suppose that there is a non-decreasing function $h=h_{\theta, \lambda}$ such that $w(\lambda, x)=$ $h(x) w(\theta, x)$. Then $y_{\theta} \leq y_{\lambda}$ and $w\left(\theta, y_{\theta}\right)=0$ imply $w\left(\lambda, y_{\theta}\right)=0$. For any $x_{1} \leq x_{2}$ we consider several cases depending on the position of $x_{1}$ and $x_{2}$ relative to $y_{\theta}$ and $y_{\lambda}$, and easily conclude that bound 2.6 holds. This shows that $L(\lambda, x)=-\log \left(w_{\lambda}(x)\right)$ is submodular, and thus completes the proof of Theorem 2.1.

\section{SeVEn ClAsses of Weight FUnCtions}

To show the encompassing nature and power of Theorem 2.1, we next present seven illustrative examples of log-supermodular functions. The first three examples correspond to the Esscher, conditional tail expectation (CTE), and the Kamps premiums, which are exceptionally well-known in the actuarial literature. The other four examples are 'mathematical inventions', but their varying convex or concave shapes hint at potential usefulness. Moreover, we have to note that the mathematical literature is not particularly generous with examples of log-supermodular functions, and thus the ones that we offer here can be viewed as contributions to the area of Mathematical Analysis and especially of Function Theory. Since the four weight functions are somewhat complex, we shall supplement their definitions with graphs.

\section{Example 3.1.}

(1) Let $w_{1}(\lambda, x)=e^{\lambda x}$. The corresponding weighted premium $H[\lambda, X]$ is known in the literature as the Esscher premium (see, e.g., Denuit et al., 2005, and references therein).

(2) Let $w_{2}(\lambda, x)=\mathbf{1}\{x>\lambda\}$. The corresponding weighted premium $H[\lambda, X]$, which can be written as $\mathbf{E}[X \mid X>\lambda]$, is known as the conditional tail expectation (CTE). We refer to Denuit et al. (2005) for detailed information on the CTE premium. 
(3) Let $w_{3}(\lambda, x)=1-e^{-x / \lambda}$. The corresponding weighted premium $H[\lambda, X]$ is known as the Kamps premium (Kamps, 1998; see also Furman and Zitikis, 2008, 2009).

(4) Let $w_{4}(\lambda, x)=e^{\left((1+x)^{\lambda}-1\right) / \lambda}-x$. The fact that $x \mapsto w_{4}(\lambda, x)$ is non-decreasing for every $\lambda>0$ is easy to establish.

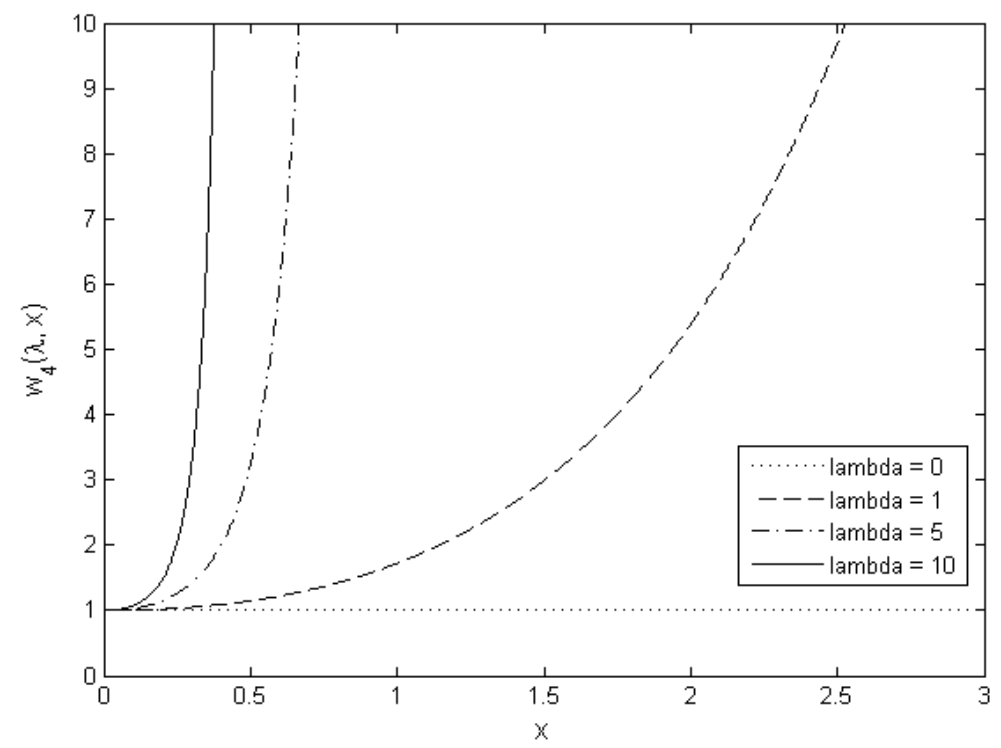

Figure 3.1. The function $x \mapsto w_{4}(\lambda, x)$. When $x \downarrow 0$, then $w_{4}(\lambda, x)$ converges to 1 for every $\lambda>0$.

(5) Let $w_{5}(\lambda, x)=\left((1+\lambda)^{x}-1\right) /(x \lambda)$. It is easy to show that $x \mapsto w_{5}(\lambda, x)$ is nondecreasing for every $\lambda>0$.

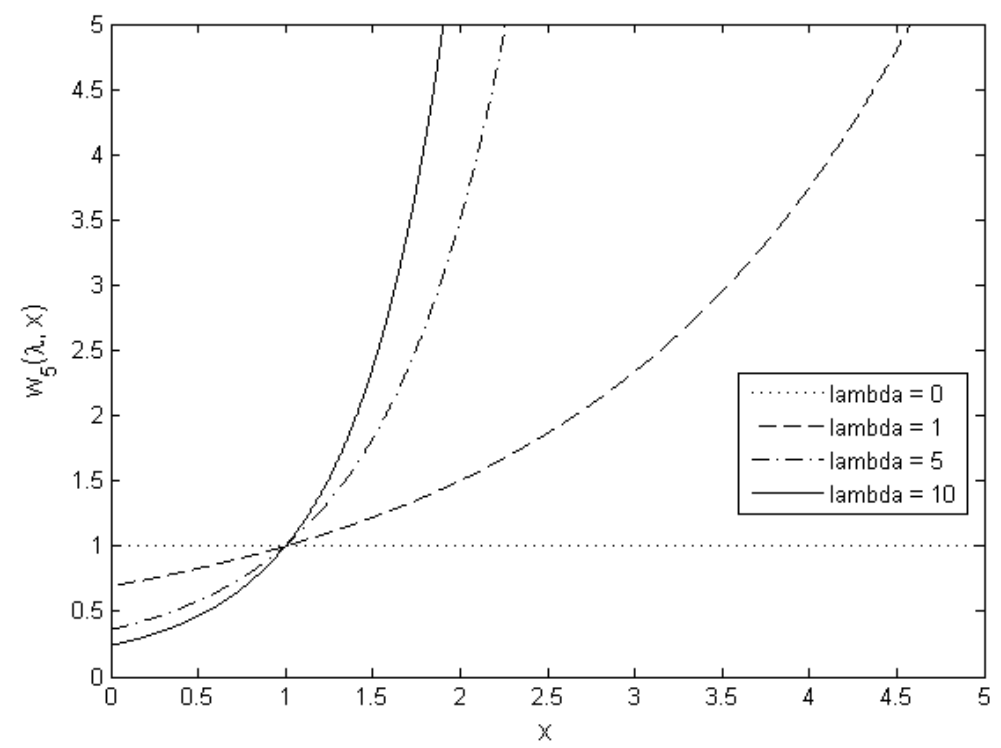

Figure 3.2. The function $x \mapsto w_{5}(\lambda, x)$. When $x \downarrow 0$, then $w_{5}(\lambda, x)$ converges to $\log (1+\lambda) / \lambda$ for every $\lambda>0$. 
(6) Let $w_{6}(\lambda, x)=(x \lambda) / \log (1+x \lambda)$. It is easy to check that $x \mapsto w_{6}(\lambda, x)$ is nondecreasing for every $\lambda>0$.

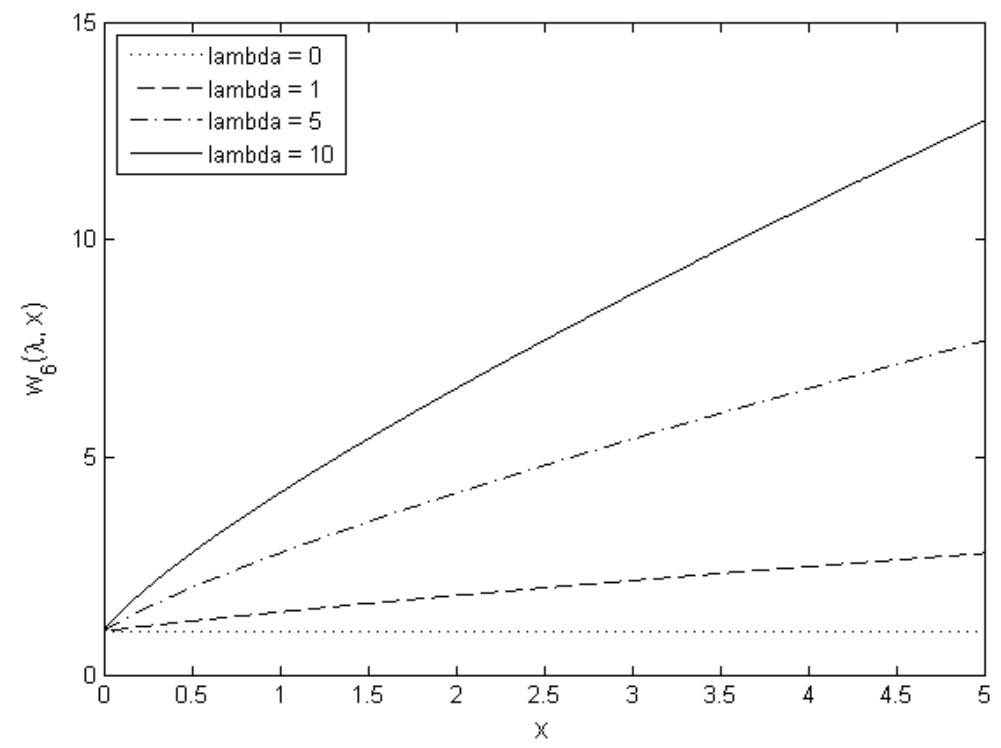

Figure 3.3. The function $x \mapsto w_{6}(\lambda, x)$. When $x \downarrow 0$, then $w_{6}(\lambda, x)$ converges to 1 for every $\lambda>0$.

(7) Let $w_{7}(\lambda, x)=\frac{\log (1+x+\lambda)}{x+\lambda} \frac{x}{\log (1+x)}$. The fact that $x \mapsto w_{7}(\lambda, x)$ is non-decreasing for every $\lambda>0$ is proved in Lemma 6.4.

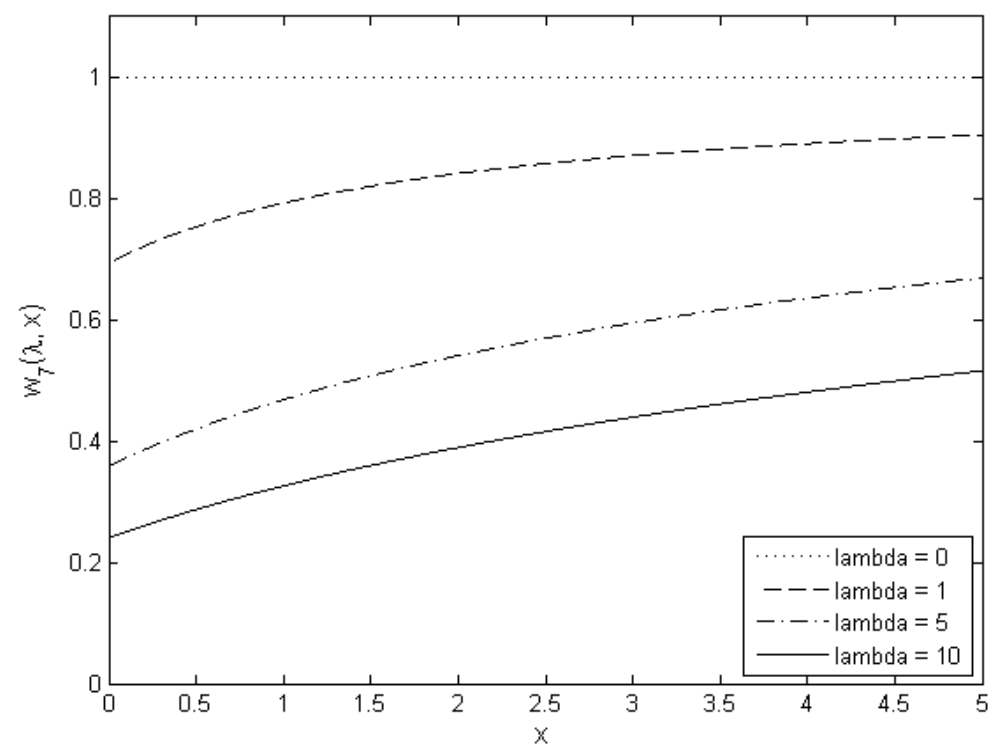

FigurE 3.4. The function $x \mapsto w_{7}(\lambda, x)$. When $x \downarrow 0$, then $w_{7}(\lambda, x)$ converges to $\log (1+\lambda) / \lambda$ (same limit as in Figure 3.2 ) for every $\lambda>0$.

From mathematical definitions and accompanying graphs, we see monotonic features of the functions $w_{i}(\lambda, x)$ with respect to $\lambda$. Specifically, for every pair $\theta<\lambda$, we check - in most cases quite easily - the following properties: 
- $w_{1}(\theta, x)<w_{1}(\lambda, x)$ for all $x \in(0, \infty)$

- $w_{2}(\theta, x) \geq w_{2}(\lambda, x)$ for all $x \in(0, \infty)$

- $w_{3}(\theta, x)>w_{3}(\lambda, x)$ for all $x \in(0, \infty)$

- $w_{4}(\theta, x)<w_{4}(\lambda, x)$ for all $x \in(0, \infty)$, which follows from the easy-to-verify inequality $\left((1+x)^{\theta}-1\right) / \theta<\left((1+x)^{\lambda}-1\right) / \lambda$

- $w_{5}(\theta, x)>w_{5}(\lambda, x)$ for all $x \in(0,1)$ $w_{5}(\theta, x)=w_{5}(\lambda, x)=1$ when $x=1$ $w_{5}(\theta, x)<w_{5}(\lambda, x)$ for all $x>1$

- $w_{6}(\theta, x)<w_{6}(\lambda, x)$ for all $x \in(0, \infty)$

- $w_{7}(\theta, x)>w_{7}(\lambda, x)$ for all $x \in(0, \infty)$; see Lemma 6.4

These monotonicity properties play important roles when establishing strict monotonicity of the corresponding functions $\lambda \mapsto H[\lambda, X]$ in Section 4 . They are also helpful and indeed decisive when determining the set $\Lambda[w, X]$ of those $\lambda>0$ for which the premium $H[\lambda, X]$ is well-defined and finite.

Certainly, in the context of the present section, it is important to check log-supermodularity of the seven functions of Example 3.1. This makes the content of the following theorem.

Theorem 3.1. The seven function $(\lambda, x) \mapsto w_{i}(\lambda, x), 1 \leq i \leq 7$, are log-supermodular.

Proof. In the case of the first two functions of Example 3.1, we shall use the noted (and proved) fact that $(\lambda, x) \mapsto w(\lambda, x)$ is log-supermodular if and only if for every pair $\theta \leq \lambda$ there is a non-decreasing function $h(x)=h_{\theta, \lambda}(x)$ such that equation (2.5) holds. In the case of the remaining five functions of Example 3.1, we shall use the fact that the function $(\lambda, x) \mapsto w(\lambda, x)$ is log-supermodular if and only if $\left(\partial^{2} / \partial \lambda \partial x\right) L(\lambda, x) \leq 0$ for all $(\lambda, x)$. Recall that $L(\lambda, x)=-\log (w(\lambda, x))$.

(1) The function $w_{1}(\lambda, x)$ is log-supermodular. This follows from decomposition 2.5 with $h(x)=e^{(\lambda-\theta) x}$, which is an increasing function whenever $\theta<\lambda$.

(2) The function $w_{2}(\lambda, x)$ is log-supermodular, which follows from decomposition (2.5) with $h(x)=\mathbf{1}\{x>\lambda\}$, which is non-decreasing.

(3) The log-supermodularly of $w_{3}(\lambda, x)$ follows from $\left(\partial^{2} / \partial \lambda \partial x\right) L(\lambda, x) \leq 0$. Indeed, since $L(\lambda, x)=-\log \left(1-e^{-x / \lambda}\right)$, we have that

$$
\frac{\partial^{2}}{\partial \lambda \partial x} L(\lambda, x)=-\frac{e^{-x / \lambda}\left(e^{-x / \lambda}-1+x / \lambda\right)}{\lambda^{2}\left(e^{-x / \lambda}-1\right)^{2}}<0 .
$$


(4) The function $w_{4}(\lambda, x)$ is log-supermodular, for which we first check the equation

$$
\begin{aligned}
- & (x+1) \lambda^{2}\left(e^{-\left((1+x)^{\lambda}-1\right) / \lambda}\right)\left(e^{\left((x+1)^{\lambda}-1\right) / \lambda}-x\right)^{2} \frac{\partial^{2}}{\partial \lambda \partial x} L(\lambda, x) \\
= & \lambda^{2}(x+1)^{\lambda} \log (x+1)\left(e^{\left((x+1)^{\lambda}-1\right) / \lambda}-x\right) \\
& +\left(\lambda(x+1)^{\lambda} \log (x+1)-(x+1)^{\lambda}+1\right)\left(1-\left((x+1)^{\lambda}-1\right) x\right) .
\end{aligned}
$$

The right-hand side of equation (3.1) is positive for all $\lambda>0$ and $x>0$. This we formulate as Lemma 6.2 and prove in Section 6.

(5) The function $w_{5}(\lambda, x)$ is log-supermodular because $\left(\partial^{2} / \partial \lambda \partial x\right) L(\lambda, x) \leq 0$, which we prove as follows:

$$
\frac{\partial^{2}}{\partial \lambda \partial x} L(\lambda, x)=\frac{(1+\lambda)^{x-1}}{\left((1+\lambda)^{x}-1\right)^{2}}\left(x \log (1+\lambda)+1-(1+\lambda)^{x}\right) .
$$

Since $x \log (1+\lambda)+1-(1+\lambda)^{x}<0$ for all $\lambda>0$ and $x>0$, this establishes the result.

(6) The function $w_{6}(\lambda, x)$ is log-supermodular because $\left(\partial^{2} / \partial \lambda \partial x\right) L(\lambda, x) \leq 0$. Indeed, since $L(\lambda, x)=-\log (x \lambda / \log (1+x \lambda))$ we have that

$$
\frac{\partial^{2}}{\partial \lambda \partial x} L(\lambda, x)=\frac{\log (1+x \lambda)-x \lambda}{(\log (1+x \lambda)(1+x \lambda))^{2}}<0 .
$$

(7) The function $w_{7}(\lambda, x)$ is log-supermodular because $\left(\partial^{2} / \partial \lambda \partial x\right) L(\lambda, x) \leq 0$, which we prove by first establishing the equation:

$$
\begin{aligned}
& -((1+x+\lambda)(x+\lambda) \log (1+x+\lambda))^{2} \frac{\partial^{2}}{\partial \lambda \partial x} L(\lambda, x) \\
& \quad=((1+x+\lambda) \log (1+x+\lambda))^{2}-(x+\lambda)^{2} \log (1+x+\lambda)-(x+\lambda)^{2} .
\end{aligned}
$$

The right-hand side of equation $(3.2)$ is positive, which we formulate as Lemma 6.3 and prove in Section 6 .

This completes the proof of Theorem 3.1 .

We now reflect upon the above proof in the context of the easily checked fact that:

- $L(\lambda, x)=\alpha f\left(\lambda^{\alpha} x\right)$ is submodular for any non-increasing and concave function $f$ and any real number $\alpha$.

Hence, submodularity of $-\log \left(w_{1}(\lambda, x)\right)$ follows by choosing $\alpha=1$ and $f(t)=-t$. Note, however, that submodularity of $-\log \left(w_{3}(\lambda, x)\right)$ does not follow from such arguments since, with $\alpha=-1$, the function $f(t)=\log \left(1-e^{-t}\right)$ is increasing, though concave. Submodularity of $-\log \left(w_{6}(\lambda, x)\right)$ does not follow from the arguments either because, with $\alpha=1$, the function $f(t)=-\log (t / \log (1+t))$ is convex, though decreasing. Finally we note that submodularity of the weight functions $-\log \left(w_{i}(\lambda, x)\right), i \in\{5,6,7\}$, does not follow from any of the constructions given in Table I on p. 312 of Topkis (1978). 


\section{LOADING STRICT-MONOTONICITY}

To prove that the function $\lambda \mapsto H[\lambda, X]$ is (strictly) increasing, we need additional assumptions.

Assumption 4.1. For every $x$, the function $\lambda \rightarrow w(\lambda, x)$ is monotonic.

The assumption does not require $\lambda \rightarrow w(\lambda, x)$ to be increasing, nor even non-decreasing, which at first sight might be somewhat surprising. However, recall the function $\lambda \mapsto w_{5}(\theta, x)$. It might be increasing, decreasing, or constant, depending on the value of $x>0$.

Our next assumption imposes a kind of strict monotonicity on the function $\lambda \rightarrow w(\lambda, x)$ by requiring, loosely speaking, that if there is a point $x$ such that the function is constant, then the point should not be 'visible', that is, the loss variable $X$ should not take on the value $x$ almost surely.

Assumption 4.2. For every pair $\theta \neq \lambda$, the set $\{x \in[0, \infty): w(\theta, x)=w(\lambda, x)\}$ has $F$-measure zero.

To work out some intuition concerning Assumption 4.2, we look at the seven functions $w_{i}(\lambda, x)$ of Example 3.1 .

- For every $x>0, w_{1}(\theta, x) \neq w_{1}(\lambda, x)$ whenever $\theta \neq \lambda$. Same holds for $w_{3}(\lambda, x)$, $w_{4}(\lambda, x)$, and $w_{6}(\lambda, x)$.

- We have $w_{2}(\theta, x)=w_{2}(\lambda, x)$ for every $x \notin(\theta, \lambda]$ when $\theta<\lambda$. This implies that Assumption 4.2 is not satisfied.

- For every $x \in(0, \infty) \backslash\{1\}$, we have $w_{5}(\theta, x) \neq w_{5}(\lambda, x)$ whenever $\theta \neq \lambda$. When $x=1$, then $w_{5}(\theta, x)=w_{5}(\lambda, x)$, and we thus need to assume $\mathbf{P}[X=1]=0$ in order to make the point $x=1$ 'invisible'. This is equivalent to assuming the continuity of $F$ at the point $x=1$.

- For every $x>0$, we have $w_{7}(\theta, x) \neq w_{7}(\lambda, x)$ whenever $\theta \neq \lambda$.

The following assumption requires, roughly speaking, the existence of a point in the closure of the half-line $(0, \infty)$ such that all the functions $x \mapsto w(\lambda, x)$ take on one and same positive value at the point.

Assumption 4.3. For every pair $(\theta, \lambda)$ there exists a point $x_{0} \in[0, \infty]$ such that $w\left(\lambda, x_{0}\right)=$ $w\left(\theta, x_{0}\right)>0$ and the functions $x \mapsto w(\lambda, x)$ and $x \mapsto w(\theta, x)$ are either both left-continuous or both right-continuous at $x_{0}$. (If $x_{0}=0$, then they have to be right-continuous, while if $x_{0}=\infty$ they have to be left-continuous.)

Even though the above assumption may look somewhat artificial, and perhaps even strange, it is nevertheless satisfied by the seven functions $w_{i}(\lambda, x)$ of Example 3.1. 
- For every $i \in\{1,4,6\}$, we have $w_{i}(\lambda, 0)=1$ for every $\lambda>0$. Thus, $x_{0}=0$. These functions $x \mapsto w_{i}(\lambda, x)$ are continuous everywhere on $(0, \infty)$.

- For every $i \in\{2,3,7\}$, we have $w_{i}(\lambda, \infty)=1$ for every $\lambda>0$. Thus, $x_{0}=\infty$. The function $x \mapsto w_{2}(\lambda, x)$ is left-continuous on $(0, \infty)$, and the functions $x \mapsto w_{i}(\lambda, x)$ for $i \in\{3,7\}$ are continuous everywhere on $(0, \infty)$.

- We have $w_{5}(\lambda, 1)=1$ for every $\lambda>0$. Thus, $x_{0}=1$. The function $x \mapsto w_{5}(\lambda, x)$ is continuous everywhere on $(0, \infty)$.

Theorem 4.1. When $(\lambda, x) \mapsto w(\lambda, x)$ is log-supermodular and Assumptions 4.144 .3 are satisfied, then $\lambda \mapsto H[\lambda, X]$ is increasing.

Proof. We prove by contradiction. Let there be $\theta<\lambda$ such that $H[\theta, X]=H[\lambda, X]$. This can be rewritten as $\mathbf{E}\left[X_{w(\theta, \cdot)}\right]=\mathbf{E}\left[X_{w(\lambda, \cdot)}\right]$. On the other hand, from bounds (2.4) we have that $F_{w(\lambda, \cdot)} \leq F_{w(\theta, \cdot)}$ for all $x \geq 0$. Hence, the cdf's $F_{w(\lambda, \cdot)}$ and $F_{w(\theta, \cdot)}$ must coincide, except possibly on a set of Lebesque measure zero. Since these functions are right-continuous, they should therefore coincide for all $x \geq 0$. In other words, we have the equation

$$
\frac{\mathbf{E}[\mathbf{1}\{X \leq x\} w(\lambda, X)]}{\mathbf{E}[w(\lambda, X)]}=\frac{\mathbf{E}[\mathbf{1}\{X \leq x\} w(\theta, X)]}{\mathbf{E}[w(\theta, X)]}
$$

for all $x \geq 0$, and thus, in turn,

$$
\frac{w(\lambda, x)}{\mathbf{E}[w(\lambda, X)]}=\frac{w(\theta, x)}{\mathbf{E}[w(\theta, X)]}
$$

for $F$-almost all $x \geq 0$. Combining the latter equation with the right- or left-continuity postulated in Assumption 4.3, we have that equation 4.2 must hold at $x=x_{0}$. Since $w\left(\lambda, x_{0}\right)=w\left(\theta, x_{0}\right)$ by Assumption 4.3 , the expectations $\mathbf{E}[w(\lambda, X)]$ and $\mathbf{E}[w(\theta, X)]$ coincide. But then equation 4.2 says that $w(\lambda, x)=w(\theta, x)$ for $F$-almost all $x \geq 0$. This contradicts Assumption 4.2 and thus finishes the proof of Theorem 4.1 .

Corollary 4.1. For every weight function $(\lambda, x) \mapsto w_{i}(\lambda, x), i \in\{1, \ldots, 7\} \backslash\{2\}$, the corresponding function $\lambda \mapsto H[\lambda, X]$ is increasing.

The above corollary excludes the weight function $w_{2}(\lambda, x)=\mathbf{1}\{x>\lambda\}$ because it does not satisfy Assumption 4.2. Nevertheless, we have the following result.

Proposition 4.1. Let $w_{2}(\lambda, x)=1\{x>\lambda\}$. If the cdf $F$ is increasing on $(0, \infty)$, then the function $\lambda \mapsto H[\lambda, X]$ is increasing.

Proof. Equation 4.1 with the weight function $w_{2}(\lambda, x)=\mathbf{1}\{x>\lambda\}$ becomes

$$
\frac{\mathbf{P}[\lambda<X \leq x]}{1-F(\lambda)}=\frac{\mathbf{P}[\theta<X \leq x]}{1-F(\theta)},
$$


where $\theta<\lambda$. Since $F$ is increasing on $(0, \infty)$, the denominators on both sides of equation (4.3) are non-zero, that is, positive. But the numerator of the left-hand side is equal to 0 for all $x \in(\theta, \lambda]$. Hence, the right-hand side should also be equal to 0 , which means that $F(x)-F(\theta)=0$ for all $x \in(\theta, \lambda]$, and thus $F(\lambda)=F(\theta)$ in particular. Since $\theta<\lambda$, the latter equation contradicts the assumption that $F$ is increasing, thus concluding the proof of Proposition 4.1 .

\section{Continuity-type Results}

The motivating actuarial problem at the end of Section 1 demonstrates the importance of establishing continuity-type results for the function $\lambda \mapsto H[\lambda, X]$. These are naturally connected with continuity-type properties of the function $\lambda \mapsto w(\lambda, x)$. In this context, we first look at the seven functions $w_{i}(\lambda, x)$ of Example 3.1 .

- The functions $\lambda \mapsto w_{i}(\lambda, x), i \in\{1, \ldots, 7\} \backslash\{2\}$, are continuous for every $x>0$.

- The function $\lambda \mapsto w_{2}(\lambda, x)$ is right-continuous for every $x>0$.

Assumption 5.1. Let $\lambda_{0}>0$ be such that $\lim _{\lambda \downarrow \lambda_{0}} w(\lambda, x)=w\left(\lambda_{0}, x\right)$ for $F$-almost all $x>0$.

All the seven functions of Example 3.1 satisfy Assumption 5.1 for every $\lambda_{0}>0$. To connect this assumption with the right-continuity of the function $\lambda \mapsto H[\lambda, X]$, we need to interchange limit and integration operations. This is taken care by the following assumption.

Assumption 5.2. Let there exist a random variable $Y \geq 0$ such that $\mathbf{E}[Y]<\infty$ and $X w_{\lambda}(X) \leq Y$ for all $\lambda$ in a (small) neighbourhood of $\lambda_{0}>0$.

The seven functions of Example 3.1 satisfy Assumption 5.2 under the following conditions:

- For $w_{i}(\lambda, x), i \in\{1,4,5,6\}$, the moment $\mathbf{E}\left[X w_{i}\left(\lambda_{0}+\epsilon, X\right)\right]$ is finite for some $\epsilon>0$.

- For $w_{i}(\lambda, x), i \in\{2,3,7\}$, the moment $\mathbf{E}[X]$ is finite.

The next theorem now becomes obvious and its proof is omitted.

Theorem 5.1. If Assumptions 5.1 and 5.2 are satisfied, then the function $\lambda \mapsto H[\lambda, X]$ is right-continuous at $\lambda_{0}$.

We next investigate the continuity of the function $\lambda \mapsto H[\lambda, X]$.

Assumption 5.3. Let $\lambda_{0}>0$ be such that $\lim _{\lambda \rightarrow \lambda_{0}} w(\lambda, x)=w\left(\lambda_{0}, x\right)$ for $F$-almost all $x>0$.

In the case of the seven functions $w_{i}(\lambda, x)$ of Example 3.1, we have the following notes:

- The functions $\lambda \mapsto w_{i}(\lambda, x), i \in\{1, \ldots, 7\} \backslash\{2\}$, are continuous for every $x>0$ and so Assumption 5.3 is satisfied for all $\lambda_{0}>0$. 
- The function $\lambda \mapsto w_{2}(\lambda, x)$ is right-continuous. Assumption 5.3 fails because the function $\lambda \mapsto w_{2}(\lambda, x)$ has a jump (of size 1 ) at the point $x$. However, if $F$ is continuous on $(0, \infty)$, then every singleton $\{x\}$ has $F$-measure zero and thus Assumption 5.3 is satisfied for every $\lambda_{0}>0$.

The proof of the next theorem is elementary and thus omitted.

Theorem 5.2. If Assumptions 5.2 and 5.3 are satisfied, then the function $\lambda \mapsto H[\lambda, X]$ is continuous at $\lambda_{0}$.

We next present an example showing that the continuity of $F$ in the case of the weight function $w_{2}(\lambda, x)$ is crucial for the function $\lambda \mapsto H[\lambda, X]$ to be continuous. Namely, let $F$ be the empirical cdf $F_{n}$ based on a sample $x_{1}, \ldots, x_{n}$. Denote the corresponding order statistics by $x_{1: n} \leq \cdots \leq x_{n: n}$. The function $\lambda \mapsto H[\lambda, X]$ is not continuous. Indeed, it is only right-continuous: for every $1 \leq i \leq n$, the function $\lambda \mapsto H[\lambda, X]$ takes on the value $\left(x_{i: n}+\cdots+x_{n: n}\right) /(n-i+1)$ when $\lambda \in\left[x_{(i-1): n}, x_{i: n}\right)$, with the notation $x_{0: n}=0$.

\section{TeChnicalities}

Here we have collected technical details that were left out from previous sections.

Lemma 6.1. We have $(y+1) \log (y+1)>y>\log (y+1)$ for every $y>0$.

We shall use Lemma 6.1 a number of times in this section. We omit its proof as it is elementary.

Lemma 6.2. The right-hand side of equation (3.1) is positive for all $\lambda>0$ and $x>0$.

Proof. With the notation $y=\left((x+1)^{\lambda}-1\right) / \lambda$, the right-hand side of equation (3.1) is positive if and only if

$$
\begin{aligned}
\lambda(\lambda y+1) \log (\lambda y+1) & \left(e^{y}-(\lambda y+1)^{1 / \lambda}+1\right) \\
+ & ((\lambda y+1) \log (\lambda y+1)-\lambda y)\left((\lambda y+1)-\lambda y(\lambda y+1)^{1 / \lambda}\right)>0 .
\end{aligned}
$$

Note that since $\lambda>0$, we have $y>0$ if and only if $x>0$. Hence, we need to show that bound (6.1) holds for all $\lambda>0$ and $y>0$. Since $(\lambda y+1) \log (\lambda y+1)>\lambda y$ (see Lemma 6.1), we have that bound (6.1) follows from

$$
(\lambda y+1) \log (\lambda y+1)\left(e^{y}-(\lambda y+1)^{1 / \lambda}\right)-((\lambda y+1) \log (\lambda y+1)-\lambda y) y(\lambda y+1)^{1 / \lambda}>0,
$$

which can be rewritten as

$$
e^{y}>(\lambda y+1)^{1 / \lambda}\left(1+y-\frac{\lambda y^{2}}{(\lambda y+1) \log (\lambda y+1)}\right) .
$$


This is an interesting bound on its own, and we shall dwell upon it once the proof of Lemma 6.2 has been finished. To prove bound $(6.2)$, we apply the logarithmic function and see that the bound is equivalent to $g(y)>0$, where

$$
g(y)=y-\frac{\log (\lambda y+1)}{\lambda}-\log \left(1+y-\frac{\lambda y^{2}}{(\lambda y+1) \log (\lambda y+1)}\right) .
$$

The function $g(y)$ converges to 0 when $y \downarrow 0$, and so to prove its positivity, we show that it is strictly increasing. For this, we first verify the equation

$$
(\lambda y+1) \log (\lambda y+1) g^{\prime}(y)=\frac{((\lambda y+1) \log (\lambda y+1)-\lambda y)\left(\log (\lambda y+1) \lambda y^{2}+\lambda y-\log (\lambda y+1)\right)}{(\lambda y+1) \log (\lambda y+1)+y((\lambda y+1) \log (\lambda y+1)-\lambda y)} .
$$

Using Lemma 6.1, we check that the numerator and denominator in the above ratio are positive. This implies that $g^{\prime}(y)>0$ and concludes the proof of Lemma 6.2.

The above proof of log-supermodularity of $w_{4}(\lambda, x)$ contains an interesting element, which is related to bound $(6.2)$. Namely, it is well-known from Calculus that, for every $y>0$, the function $\lambda \mapsto(\lambda y+1)^{1 / \lambda}$ is decreasing and $\lim _{\lambda \downarrow 0}(\lambda y+1)^{1 / \lambda}=e^{y}$. With the establishment of bound 6.2), we have obtained a lower bound for the relative error of this approximation of $e^{y}$. Namely, we have that

$$
\frac{e^{y}-(\lambda y+1)^{1 / \lambda}}{(\lambda y+1)^{1 / \lambda}}>y\left(1-\frac{\lambda y}{(\lambda y+1) \log (\lambda y+1)}\right)>0
$$

for all $\lambda>0$ and $y>0$.

Lemma 6.3. The right-hand side of equation (3.2) is positive for all $\lambda>0$ and $x>0$.

Proof. Substituting $y=x+\lambda$, we obtain that the expression on the right-hand side of equation 3.2 is positive if and only if $f(y)>0$, where

$$
f(y)=((1+y) \log (1+y))^{2}-y^{2} \log (1+y)-y^{2} .
$$

Since $f(y)=0$ when $y=0$, we have $f(y)>0$ for all $y>0$ if the function $f$ is strictly increasing. For this, we check the equation $(1+y) f^{\prime}(y)=g(y)$, where

$$
g(y)=2((1+y) \log (1+y))^{2}-3 y^{2}+2(1+y) \log (1+y)-2 y .
$$

To show that $g(y)>0$ for all $y>0$, we note that $g(0)=0$ and then show that $g$ is strictly increasing. For this, we check the equation

$$
(1+y) g^{\prime}(y)=4((1+y) \log (1+y))^{2}+4 y(1+y) \log (1+y)-6 y^{2}+6(1+y) \log (1+y)-6 y .
$$

The right-hand side of the equation is positive for all $y>0$, which follows from the bound $(y+1) \log (y+1)>y$ (see Lemma 6.1). This concludes the proof of Lemma 6.3.

Lemma 6.4. The function $(\lambda, x) \mapsto w_{7}(\lambda, x)$ is increasing in $x$ and decreasing in $\lambda$. 
Proof. First, a simple calculation shows that

$$
\begin{aligned}
(1+x)(1+x+\lambda)(x+\lambda)^{2}(\log (1+x))^{2} \frac{\partial}{\partial x} w_{7}(\lambda, x) \\
=x(1+x)(x+\lambda) \log (1+x)+\lambda(1+x)(1+x+\lambda) \log (1+x) \log (1+x+\lambda) \\
\quad-x(x+\lambda)(1+x+\lambda) \log (1+x+\lambda) .
\end{aligned}
$$

From this equation we conclude that

$$
\begin{aligned}
& \lim _{\lambda \rightarrow 0} \frac{\partial}{\partial x} w_{7}(\lambda, x)=0, \\
& \lim _{x \rightarrow 0} \frac{\partial}{\partial x} w_{7}(\lambda, x)=\frac{2 \lambda-\lambda \log (1+\lambda)-2 \log (1+\lambda)+\lambda^{2} \log (1+\lambda)}{2 \lambda^{2}(1+\lambda)}>0 .
\end{aligned}
$$

We have shown in Lemma 6.3 that $\left(\partial^{2} / \partial \lambda \partial x\right) \log \left(w_{7}(\lambda, x)\right)>0$, which can be rewritten as

$$
\frac{\partial}{\partial \lambda}\left(\frac{(\partial / \partial x) w_{7}(\lambda, x)}{w_{7}(\lambda, x)}\right)>0,
$$

thus implying that the function $\lambda \mapsto\left((\partial / \partial x) w_{7}(\lambda, x)\right) / w_{7}(\lambda, x)$ is strictly increasing. But the function takes on the value 0 at $\lambda=0$, and thus the function is positive for all $x>0$ and $\lambda>0$. Since the denominator $w_{7}(\lambda, x)$ is strictly positive, we have $(\partial / \partial x) w_{7}(\lambda, x)>0$ for all $x>0$ and $\lambda>0$. Consequently, $w_{7}(\lambda, x)$ is increasing in $x$.

To show that $w_{7}(\lambda, x)$ is decreasing in $\lambda$, we check the equation

$$
(1+x+\lambda)(x+\lambda)^{2} \log (1+x) \frac{\partial}{\partial \lambda} w_{7}(\lambda, x)=x(x+\lambda-(1+x+\lambda) \log (1+x+\lambda)) .
$$

Lemma 6.1 implies that the right-hand side of equation 6.4 is negative. This concludes the proof of Lemma 6.4.

\section{ACKNOWLEDGMENTS}

The research was partially supported by the Natural Sciences and Engineering Research Council (NSERC) of Canada.

\section{REFERENCES}

Broll, U., Egozcue, M., Wong, W.K. and Zitikis, R.(2010). Prospect theory, indifference curves, and hedging risks. Applied Mathematics Research Express (to appear).

Denuit, M., Dhaene, J., Goovaerts, M.J., Kaas, R., 2005. Actuarial Theory for Dependent Risk: Measures, Orders and Models. Wiley, New York.

Denneberg, D. (1994). Non-Additive Measure and Integral. Kluwer, Dordrecht.

Egozcue, M., Fuentes García, L., Wong, W.K. and Zitikis, R. (2010). The covariance sign of transformed random variables with applications to economics and finance. IMA Journal of Management Mathematics (to appear).

Fujishige, S. (1991). Submodular Functions and Optimization. North-Holland, Amsterdam. 
Furman, E. and Zitikis, R. (2008). Weighted premium calculation principles. Insurance: Mathematics and Economics, 42, 459-465.

Furman, E. and Zitikis, R. (2009). Weighted pricing functionals with applications to insurance: an overview. North American Actuarial Journal, 13, 483-496.

Jones, B.L. and Zitikis, R. (2007). Risk measures, distortion parameters, and their empirical estimation. Insurance: Mathematics and Economics, 41, 279-297.

Kamps, U. (1998). On a class of premium principles including the Esscher principle. Scandinavian Actuarial Journal, 1998, 75-80.

Lehmann, E.L. (1966). Some concepts of dependence. Annals of Mathematical Statistics, 37, 1137-1153.

Mitrinović, D.S., Pečarić, J.E. and Fink, A.M. (1993). Classical and New Inequalities in Analysis. Kluwer, Dordrecht.

Narayanan, H. (1997). Submodular Functions and Electrical Networks. North-Holland, Amsterdam.

Pečarić, J.E., Proschan, F. and Tong, Y.L. (1992). Convex Functions, Partial Orderings, and Statistical Applications. Academic Press, Boston.

Topkis, D.M. (1978). Minimizing a submodular function on a lattice. Operations Research, 26, 305-321.

Topkis, D.M. (2001). Supermodularity and Complementarity. Princeton University Press, Princeton. 